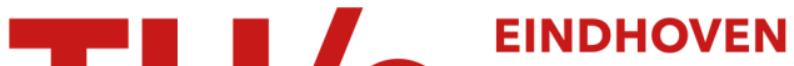 UNIVERSITY OF TECHNOLOGY
}

\section{High-brightness, compact soft $x$-ray source based on Cherenkov radiation}

\section{Citation for published version (APA):}

Knulst, W., van der Wiel, M. J., Luiten, J., \& Verhoeven, J. (2004). High-brightness, compact soft x-ray source based on Cherenkov radiation. In G. A. Kyrala, J-C. J. Gauthier, C. A. MacDonald, \& A. M. Khousary (Eds.), Laser-Generated and Other Laboratory X-Ray and EUV Sources, Optics, and Applications (pp. 393-404). (Proceedings of SPIE - The International Society for Optical Engineering; Vol. 5196). SPIE. https://doi.org/10.1117/12.516144

DOI:

10.1117/12.516144

Document status and date:

Published: 03/05/2004

\section{Document Version:}

Publisher's PDF, also known as Version of Record (includes final page, issue and volume numbers)

\section{Please check the document version of this publication:}

- A submitted manuscript is the version of the article upon submission and before peer-review. There can be important differences between the submitted version and the official published version of record. People interested in the research are advised to contact the author for the final version of the publication, or visit the $\mathrm{DOI}$ to the publisher's website.

- The final author version and the galley proof are versions of the publication after peer review.

- The final published version features the final layout of the paper including the volume, issue and page numbers.

Link to publication

\section{General rights}

Copyright and moral rights for the publications made accessible in the public portal are retained by the authors and/or other copyright owners and it is a condition of accessing publications that users recognise and abide by the legal requirements associated with these rights.

- Users may download and print one copy of any publication from the public portal for the purpose of private study or research.

- You may not further distribute the material or use it for any profit-making activity or commercial gain

- You may freely distribute the URL identifying the publication in the public portal.

If the publication is distributed under the terms of Article $25 \mathrm{fa}$ of the Dutch Copyright Act, indicated by the "Taverne" license above, please follow below link for the End User Agreement:

www.tue.nl/taverne

Take down policy

If you believe that this document breaches copyright please contact us at:

openaccess@tue.nl

providing details and we will investigate your claim. 


\section{High-brightness compact soft x-ray source based on Cherenkov radiation}

Walter Knulst, Marnix J. van der Wiel, O. Jom Luiten, Jan Verhoeven

Walter Knulst, Marnix J. van der Wiel, O. Jom Luiten, Jan Verhoeven, "Highbrightness compact soft x-ray source based on Cherenkov radiation," Proc. SPIE 5196, Laser-Generated and Other Laboratory X-Ray and EUV Sources, Optics, and Applications, (7 January 2004); doi: 10.1117/12.516144

Event: Optical Science and Technology, SPIE's 48th Annual Meeting, 2003, San Diego, California, United States 


\title{
High-brightness, compact soft x-ray source based on Cherenkov radiation
}

\author{
Walter Knulst ${ }^{*}$, Marnix J. van der Wiel ${ }^{\mathrm{a}}$, Jom Luiten ${ }^{\mathrm{a}}$, Jan Verhoeven ${ }^{\mathrm{b}}$ \\ ${ }^{\mathrm{a}}$ Eindhoven University of Technology, Department of Applied Physics, P.O. Box 513, $5600 \mathrm{MB}$ \\ Eindhoven, The Netherlands; \\ ${ }^{\mathrm{b}}$ FOM Institute for Atomic and Molecular Physics, Kruislaan 407, 1098 SJ Amsterdam, The \\ Netherlands
}

\begin{abstract}
Cherenkov radiation in the soft x-ray region is generated in narrowband regions at inner-shell absorption edges. Mainly low-Z elements are suitable Cherenkov sources, which emit in a photon energy range from $30 \mathrm{eV}$ to $1 \mathrm{keV}$ and require moderate electron energies up to $25 \mathrm{MeV}$. Generally, in the soft x-ray region materials are highly absorbing and therefore the Cherenkov radiation theory is discussed for absorbing media. A detailed description includes transition radiation that is generated at the interface when the relativistic electron exits the material. We show that the transition radiation yield equation, when it is adopted for an absorbing medium, includes Cherenkov radiation. Based on this approach it is shown that the spectral intensity of Cherenkov radiation in the soft X-ray region is large compared to transition radiation for moderate electron energies. First measurements of soft x-ray Cherenkov radiation in the waterwindow spectral region, generated in titanium and vanadium foils, are discussed in detail. The measured spectral and angular distribution of the radiation, and the measured total yield $\left(\approx 10^{-4}\right.$ photon per electron $)$ are in agreement with theoretical predictions based on the refractive index data. We show that the brightness that can be achieved using a small electron accelerator is sufficient for practical $\mathrm{x}$-ray microscopy in the water window.
\end{abstract}

Keywords: Complex dielectric constant, Cherenkov radiation, transition radiation, soft x-ray source, relativistic electrons

\section{INTRODUCTION}

Recently, interest has grown in compact (laboratory-sized) high-brightness soft x-ray sources ${ }^{1}$. So far, the highest quality of intense soft x-ray light, which is expressed in the quantity brightness (intensity per solid angle per bandwidth per unit source area), is obtained from undulators in storage rings. Therefore, applications of these light sources, for instance $\mathrm{x}$ ray microscopy, $\mathrm{x}$-ray crystallography, $\mathrm{x}$-ray diffraction and fluorescence $\mathrm{x}$-ray microprobes, are limited to these synchrotron light facilities. Although small-sized soft x-ray sources may never reach the brightness level of undulator radiation, they are certainly interesting for making synchrotron experimental techniques available at smaller institutes.

Over the last decade, the efficiency of plasma-based soft x-ray sources has improved: (i) pinch plasmas, in which a high current pulse is sent through a plasma to make it collapse, (ii) laser-produced plasma ${ }^{2,3}$, in which high-power laser pulses are fired at liquid jets or droplets, (iii) high-harmonic generation with femtosecond laser pulses ${ }^{4,5}$, in which very short laser pulses are sent through a gas jet. The first two sources produce line emission spectra from highly ionized atoms such as $\mathrm{C}, \mathrm{N}, \mathrm{O}$ or $\mathrm{Xe}$. As a side effect these sources produce a certain amount of debris depending on the target material. The third source produces odd harmonics of the fundamental wavelength of a high-intensity laser pulse by a non-linear process in the gas jet, but these harmonics tend to merge into a continuous spectrum when approaching the carbon K-edge $(284 \mathrm{eV})$. Recently, it has been shown that laser-produced plasmas ${ }^{2}$ and high-harmonic generation ${ }^{6}$ are $^{2}$ sufficiently bright for practical imaging applications.

Compact electron-accelerator-based sources ${ }^{7}$ have received relatively little attention up till now. Among interactions of relativistic electrons with a medium that cause emission of radiation, such as (resonant) transition radiation, channeling radiation and parametric $\mathrm{x}$-rays, especially Cherenkov radiation is a promising candidate for a compact soft x-ray source. As we will show in this paper, in the soft x-ray region the Cherenkov radiation is characterized by a single-line spectrum and by forwardly directed emission and only requires low-relativistic electrons from a laboratory-sized accelerator. For a long time, Cherenkov radiation was excluded in the soft x-ray region, because at these wavelengths materials are highly

"w.knulst@tue.nl; phone +31-40-2474030; fax +31-40-2438060; www.tue.nl

Laser-Generated \& Other Lab. X-Ray \& EUV Sources, Optics, Apps., ed. G. A. Kyrala,

J.-C. J. Gauthier, C. A. MacDonald, A. M. Khounsary, Proceedings of SPIE Vol. 5196

(SPIE, Bellingham, WA, 2004) $\cdot 0277-786$ X/04/\$15 $\cdot$ doi: 10.1117/12.516144 
absorbing and the refractive index is generally smaller than unity. Bazylev et al. ${ }^{8}$, however, realized that at some innershell absorption edges the refractive index exceeds unity and Cherenkov radiation will be generated in a narrowband region, which was demonstrated for the carbon K-edge by using 1.2-GeV electrons. Two years ago we demonstrated ${ }^{9}$, by generating Cherenkov radiation at the silicon L-edge, that low-relativistic electrons are already sufficient for a reasonable yield.

In section 2 of this paper we present a detailed theoretical analysis of soft x-ray Cherenkov radiation from absorbing materials. In section 2.2 the resonant behaviour in the refractive index, which enables Cherenkov radiation in the soft xray region, is discussed. In section 2.3 we present a simple theoretical approach for the Cherenkov yield in the soft $\mathrm{x}$-ray region. Absorption is taken into account by an absorption factor that is introduced into the spectral Cherenkov yield equation for a transparent medium. To check the validity of this approach we present in section 2.4 a full description by taking also transition radiation into account, which is generated by the relativistic electron at the interface between the medium and vacuum. In this case absorption is introduced by substituting the complex refractive index into the spectral transition radiation equation for a transparent medium. As a consequence Cherenkov radiation for an absorbing medium is included and is in approximation equal to the simple approach as will be shown in section 2.4.

Based on the theoretical predictions in section 2, we have experimentally analyzed the Cherenkov radiation yield of two of these materials, namely $\mathrm{Ti}$ and $\mathrm{V}$, which will be presented in detail in section 3 . These measurements are the first observations of soft $\mathrm{x}$-ray Cherenkov radiation generated within the water-window spectral region. In section 4 the Cherenkov source will be compared to other high-brightness, compact soft x-ray sources.

\section{SOFT X-RAY CHERENKOV RADIATION}

\subsection{Cherenkov condition}

Cherenkov radiation is emitted by a charged particle that travels through a medium with refractive index $n$, when its velocity $v$ exceeds the phase velocity of light $c / n$. This 'shock wave of light' is emitted at the angle of constructive interference, given by

$$
\cos \theta=\frac{c / n}{v}
$$

Therefore, Cherenkov light will be emitted by relativistic electrons $(v \approx c)$, which are sent through a foil, if the refractive index of the material exceeds unity $(n>c / v \approx 1)$. Due to the fact that materials are dispersive, Cherenkov radiation can

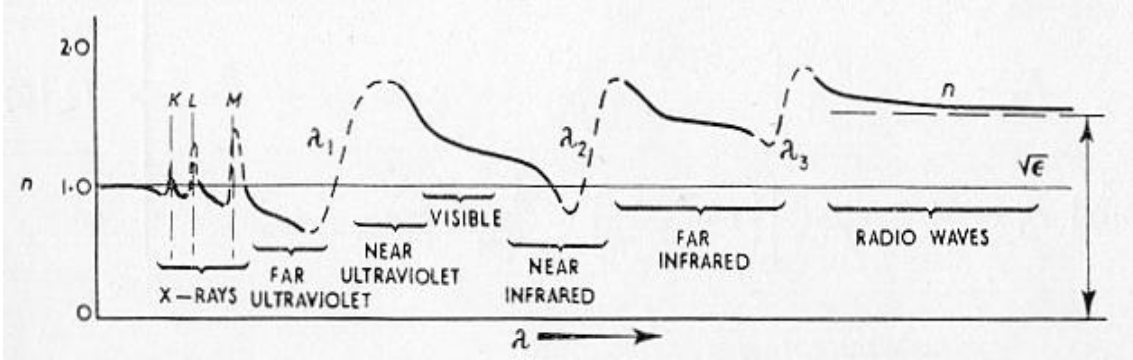

Figure 1: General behaviour of the refractive index of optically transparent materials ${ }^{11}$.

only be generated in limited spectral regions (see fig. 1). In the visible region Cherenkov radiation is a well-known effect, where it is often applied in high-energy particle detection ${ }^{10}$. In the soft $\mathrm{x}$-ray region, however, the refractive index is generally smaller than unity. The exception is at absorption edges associated with the binding energies of innerelectron shells, where the refractive index may exceed unity due to resonant anomalous dispersion.

\subsection{Refractive index in the soft $x$-ray region}

In the soft x-ray region all materials are highly absorbing and the refractive index is therefore a complex number $(\tilde{n}=n+i k)$. The real part of the refractive index $(n=1-\delta)$ describes the phase propagation of waves, while the imaginary part $(k)$ represents the absorption. Typically, for soft x-ray and higher photon energies the values of $\delta$ and $k$ 
are in the same order of magnitude and much smaller than unity $(|\delta|, k<<1)$. In this regime it is more convenient to use the complex susceptibility $\left(\chi=\chi^{\prime}+i \chi^{\prime \prime}\right)$, which is related to the refractive index by

$$
\tilde{n}^{2}=\varepsilon=1+\chi
$$

with $\varepsilon$ the complex dielectric constant.

Generally, the real part of the dielectric constant is smaller than unity for photon energies above the valence-electron plasma frequency $\omega_{p}$ (typically $\hbar \omega=10-20 \mathrm{eV}$ ). In this spectral region the dispersion of a medium can be described by a simple model ${ }^{12}$ and at frequencies much higher than binding frequencies (i.e. binging energies of inner-electron shells) the dielectric constant is approximated by

$$
\operatorname{Re}(\varepsilon)=1+\chi^{\prime}=1-\frac{\omega_{p}^{2}}{\omega^{2}}
$$

in which the plasma frequency $\omega_{p}$ is defined by

$$
\omega_{p}^{2}=\frac{N_{e} e^{2}}{\varepsilon_{0} m_{e}}
$$

with $m_{e}$ the electron mass and $N_{e}$ is the density of electrons with a binding energy smaller than $\hbar \omega$. In equation (3) the frequency $\omega_{p}$ changes in steps every time an inner-electron shell is passed for increasing photon energy. This general behaviour is often used to calculate the transition radiation power in the x-ray region, as will be discussed in section 2.3.

Close to absorption edges this simple plasma description is not adequate. At these photon energies the real part of the dielectric constant shows anomalous resonant dispersion. Such a resonance can be so strong that the real part of the susceptibility is positive, i.e. the real part of the refractive index is larger than unity. In these narrow spectral regions Cherenkov radiation can be generated as Bazylev et al. ${ }^{8}$ realized. In a thorough analysis by Smith et al. ${ }^{13}$ is discussed which elements will preferably show this strong resonance behaviour. First, the most likely candidates are $\mathrm{L}_{\mathrm{IIIIII}}{ }^{-}$and $\mathrm{M}_{\mathrm{IV}, \mathrm{V}}$-edges involving shells containing a large number of electrons. Exceptions are the K-edges of second-period elements, because these lack a well-developed L-shell. Additionally, the sub-shells in such a shell should not be separated too much in energy, which is the case for low-Z elements. Second, the edges must be sufficiently isolated in energy and must have a large jump in absorption. These requirements do not favor 3-d transition metals with broad Mshell absorption bands.

Taking the above predictions for favorable elements into account and looking into the refractive index data of Henke et $a l .{ }^{14}$ it appears that solid-state materials with an atomic number lower than about 30 will have the ability to generate line-like Cherenkov radiation at either their K-edge (second period) or $\mathrm{L}_{\mathrm{III}}$-edge (third and fourth period). These photon energies lie all within the soft x-ray region $(30 \mathrm{eV}$ to $1 \mathrm{keV})$ and can be generated by electrons of moderate energy (lower than $25 \mathrm{MeV}$ ). In the data of ref. 14 the refractive index of more high-Z elements (e.q. Fe, $\mathrm{Cr}$ at the M-edge) will also exceed unity, but these resonances are much broader and are therefore not suitable for narrowband soft x-ray Cherenkov sources. In fig. 3a and fig. 3b examples are shown of the complex susceptibility of silicon and titanium at the L-edge. The dashed line indicates the Cherenkov condition. Notice that the total number of data points of ref. 14 exceeding unity at the absorption edge is very limited.

\subsection{Soft x-ray Cherenkov radiation described by the Frank-Tamm equation}

In this section the properties and intensity of soft x-ray Cherenkov radiation generated in an absorbing medium are described by a simple approach. The analysis is started from the Frank-Tamm equation ${ }^{11,15}$ (6), which is derived from Maxwell's equations and describes the spectral Cherenkov intensity emitted by a charged particle per unit length in a non-absorbing medium (real index of refraction) integrated over all emission angles.

$$
\frac{d^{2} W}{d l d \omega}=\frac{q^{2}}{4 \pi \varepsilon_{0} c^{2}}\left(1-\frac{1}{\beta^{2} n^{2}}\right) \omega
$$

with $\beta=v / c$ the relative velocity of the charged particle. In the soft $\mathrm{x}$-ray region $(n \approx 1)$ and for relativistic electrons $\left(\gamma=\left(1-\beta^{2}\right)^{-1 / 2} \gg 1\right)$ this expression can be approximated and expressed in terms of susceptibility. The spectral yield (number of photons per frequency) per unit length is given by 


$$
\frac{d^{2} N}{d l d \omega}=\frac{\alpha}{c}\left(\chi^{\prime}-\gamma^{-2}\right)
$$

with $\alpha \approx 1 / 137$ the fine structure constant. In this equation (7) the Cherenkov condition is expressed by $\chi^{\prime}-\gamma^{-2}>0$. The total yield (photons per electron) is proportional to the area under the curve $\chi^{\prime}-\gamma^{-2}$ and to the path length through the medium. However, the latter does not hold for an absorbing medium, where the generated photons can be absorbed in the medium before reaching the surface. Therefore, the intensity exiting the material from a depth $l$ in the material is the intensity of equation (7) times an exponential decay factor characterized by the absorption length $l_{a b s}$.

$$
\frac{d^{2} N}{d l d \omega}=\frac{\alpha}{c}\left(\chi^{\prime}-\gamma^{-2}\right) e^{-\frac{l}{l_{a b s}}}
$$

The absorption length of a material is given by

$$
l_{a b s} \approx \frac{c}{\omega \chi^{\prime \prime}}
$$

Integrating equation (8) over a thickness that is larger than the absorption length gives the spectral Cherenkov yield for an absorbing medium,

$$
\frac{d N}{d \omega}=\frac{\alpha}{\omega}\left(\frac{\chi^{\prime}-\gamma^{-2}}{\chi^{\prime \prime}}\right)
$$

Equation (10) shows that a high spectral Cherenkov yield from an absorbing medium is obtained when $\chi^{\prime}-\gamma^{-2}>\chi^{\prime \prime}$. Taking a look at the resonant anomalous dispersion this is mainly fulfilled at the non-absorbing site of an absorption edge. In fig. $2 \mathrm{~b}$ the spectral yield is calculated for silicon L-edge Cherenkov radiation generated by $10-\mathrm{MeV}$ electrons using the equation (10). Clearly is seen that on the non-absorbing site of the L-edge (i.e. for photon energies slightly lower than $99.7 \mathrm{eV}$ ) the spectral yield is large.

\subsection{Soft x-ray Cherenkov radiation described by the Ginzburg-Frank equation}

As described in the previous section a relativistic electron that passes through a foil with a refractive index larger than unity will generate Cherenkov radiation along its trajectory inside the foil. This is not a complete description, because additionally, at both interfaces, entering and exiting the foil, the electron experiences a change in refractive index and as a result it will also emit transition radiation. The transition radiation spectrum in the soft x-ray region is broadband, whereas Cherenkov radiation is only generated in narrowband regions. For a foil with a thickness several times larger than the absorption length only the last interface has to be considered, because radiation produced at the first interface is fully absorbed. As will be shown in this section the resulting equation for the transition radiation yield will also contain the Cherenkov radiation yield, which is in approximation equal to the simple approach in the previous section. For very thin foils, which are not optically thick, a more thorough description ${ }^{16}$ of transition radiation is needed in which also reflections at interfaces have to be taken into account.

The analysis in this section is started from the Ginzburg-Frank equation ${ }^{17,18}$ describing transition radiation by solving Maxwell's equations with boundary condition at the interface of two transparent media. Through this interface a relativistic electron moves from medium 1 (left hand site) with refractive index $\varepsilon_{1}=\varepsilon$ into medium 2 (right hand site), which is in this case vacuum $\left(\varepsilon_{2}=1\right)$. The spectral angular yield of transition radiation emitted by the electron into vacuum with angle $\theta_{2}$ is given by

$$
\frac{d^{2} N}{d \omega d \Omega}=\frac{\alpha}{\omega} \frac{\beta^{2}}{\pi^{2}} \frac{|\varepsilon-1|^{2} \sin ^{2} \theta_{2} \cos ^{2} \theta_{2}}{\left|\varepsilon \cos \theta_{2}+\sqrt{\varepsilon-\sin ^{2} \theta_{2}}\right|^{2}} \times \frac{\left|1-\beta^{2}-\beta \sqrt{\varepsilon-\sin ^{2} \theta_{2}}\right|^{2}}{\mid\left[1-\beta^{2} \cos ^{2} \theta_{2}\right]\left[1-\left.\beta \sqrt{\varepsilon-\sin ^{2} \theta_{2}}\right|^{2}\right.}
$$

In the soft $x$-ray region $(\varepsilon \approx 1)$, for relativistic electrons $(\gamma>>1)$ and for small angles $\left(\theta_{2}<<1\right)$ equation $(11)$ can be approximated ${ }^{18}$ by

$$
\frac{d^{2} N}{d \omega d \Omega}=\frac{\alpha}{\omega} \frac{\theta_{2}^{2}}{\pi^{2}}\left|\frac{1}{-\gamma^{-2}-\theta_{2}^{2}}-\frac{1}{\chi^{\prime}-\gamma^{-2}-\theta_{2}^{2}}\right|^{2}
$$

This equation is usually applied in the x-ray region to calculate the transition radiation yield, in which the general plasma behaviour of the refractive index (3) is substituted ${ }^{17,18}$. Whereas, using the best-known refractive index data from 
ref. 14, for some photon energy one factor in the denominator in equation (11) and (12) is equal to zero if the Cherenkov condition is fulfilled in the medium. This can be shown by substituting the Snell's law of refraction $\left(\sqrt{\varepsilon} \sin \theta_{1}=\sin \theta_{2}\right)$ in the term $1-\beta \sqrt{\varepsilon-\sin ^{2} \theta_{2}}$ in the denominator of equation (11),

$$
1-\beta \sqrt{\varepsilon} \cos \theta_{1}
$$

Putting this equal to zero gives the Cherenkov angular relation in the medium with emission angle $\theta_{1}$. The same holds for the term $\chi^{\prime}-\gamma^{-2}-\theta_{2}{ }^{2}$ in the dominator in equation (12) by substituting $\theta_{1} \approx \theta_{2}$,

$$
\chi^{\prime}-\gamma^{-2}-\theta_{1}^{2}
$$

Putting this also equal to zero gives the Cherenkov angular relation in the approximation. As a consequence the transition radiation yield becomes infinite at this angle, because a half-infinite transparent medium is assumed in equation (11) and (12), which makes the generation length of Cherenkov radiation infinite. In the soft x-ray region materials are absorbing and therefore the complex dielectrical constant $\varepsilon$ or complex susceptibility $\chi$ has to be substituted. Then, the Cherenkov factor in denominator becomes purely imaginary and as a consequence the transition radiation yield stays finite. This intensity peak is identified as Cherenkov radiation from an absorbing medium. From the angular radiation profile the distinction between the two phenomena can be made. For example, in fig. $2 \mathrm{a}$ the angular emission profile is plotted for 99.7-eV radiation generated in a silicon foil for several electron energies. Transition radiation has its maximum intensity at an angle of about $\gamma^{-1}$, which therefore decreases for increasing electron energy. Cherenkov radiation on the other hand has its maximum intensity at the angle given by equation (1), which goes to a constant value for increasing electron energy. For specific electron energies the angle of transition radiation $\gamma^{-1}$ and the Cherenkov angle are equal. At this electron energy the two radiation phenomena are indistinguishable.
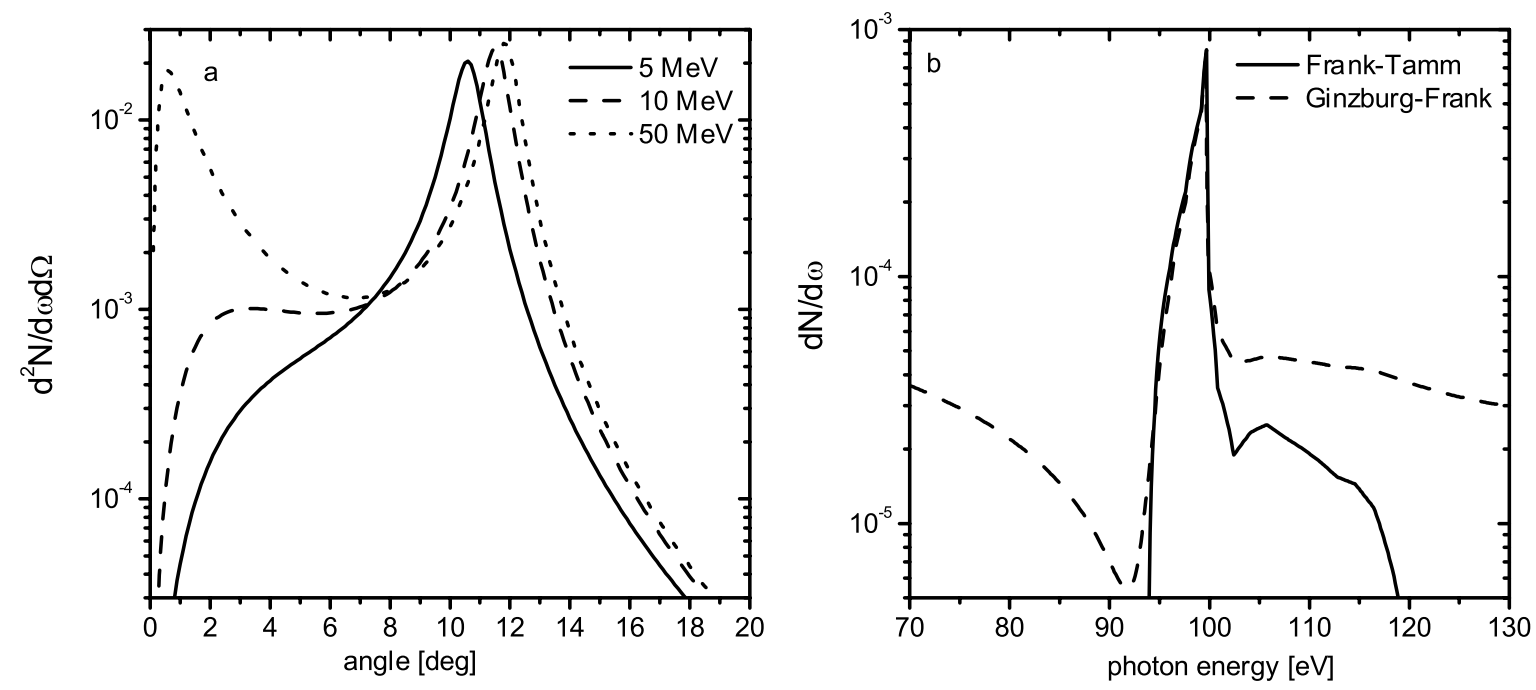

Figure 2: (a) Spectral angular yield plotted for 5, 10 and 50-MeV electron energies. Refractive index data taken from silicon at $\hbar \omega=$ $99.7 \mathrm{eV}$. (b) Specral yield from silicon for 10-MeV electrons plotted for the Frank-Tamm equation (10) and Ginzburg-Frank equation (17).

To compare this soft $\mathrm{x}$-ray transition radiation yield, which includes Cherenkov radiation, with the description of soft $\mathrm{x}$ ray Cherenkov radiation in the previous section the spectral angular yield of equation (12) has to be integrated over all emission angles. The total spectral yield is given by the integration

$$
\frac{d N}{d \omega}=2 \pi \frac{\alpha}{\omega \pi^{2}} \int_{0}^{\infty} \theta^{2}|A(\omega, \theta)|^{2} \theta d \theta
$$


with

$$
|A(\omega, \theta)|^{2}=\frac{\chi^{\prime 2}+\chi^{\prime \prime 2}}{\left(-\gamma^{-2}-\theta^{2}\right)^{2}\left(\left(\chi^{\prime}-\gamma^{-2}-\theta^{2}\right)^{2}+\chi^{\prime \prime 2}\right)}
$$

The resulting spectral yield is

$$
\frac{d N}{d \omega}=\frac{\alpha}{\omega \pi}\left\{\left[\frac{1}{2}-\frac{\gamma^{-2} \chi^{\prime}}{|\tilde{\chi}|^{2}}\right] \ln \left(\frac{\left(\chi^{\prime}-\gamma^{-2}\right)^{2}+\chi^{\prime \prime 2}}{\gamma^{-4}}\right)-1+\frac{1}{\chi^{\prime \prime}}\left[\chi^{\prime}-\gamma^{-2} \frac{\chi^{\prime 2}-\chi^{\prime \prime 2}}{|\tilde{\chi}|^{2}}\right]\left(\tan ^{-1}\left(\frac{\chi^{\prime}-\gamma^{-2}}{\chi^{\prime \prime}}\right)+\frac{\pi}{2}\right)\right\}
$$

Two parts are recognized in this expression for the spectral yield. The first part is identified as transition radiation and the second part is identified as Cherenkov radiation. The Cherenkov condition is described by the inverse tangent, with the Cherenkov condition $\chi^{\prime}-\gamma^{-2}$ as argument. When this value is much larger than zero ( $\chi$ " is by definition positive), the factor containing the inverse tangent approaches unity and if this value is much smaller zero it approaches zero. For the condition that $\chi^{\prime}-\gamma^{-2} \gg \chi^{\prime}$ "the factor containing the inverse tangent can be replaced by a Heaviside step function $(\eta)$ with the Cherenkov condition $\chi^{\prime}-\gamma^{-2}$ as argument. In this approximation equation (17) becomes

$$
\frac{d N}{d \omega}=\frac{\alpha}{\omega \pi}\left\{\left[\frac{1}{2}-\frac{\gamma^{-2}}{\chi^{\prime}}\right] \ln \left(\frac{\left(\chi^{\prime}-\gamma^{-2}\right)^{2}}{\gamma^{-4}}\right)-1\right\}+\frac{\alpha}{\omega} \frac{\chi^{\prime}-\gamma^{-2}}{\chi^{\prime \prime}} \eta\left(\chi^{\prime}-\gamma^{-2}\right)
$$

In this case the second part is equal to the Cherenkov yield for an absorbing medium described by equation (10). Therefore, on the non-absorbing site of the absorption edge, where $\chi^{\prime}-\gamma^{-2}>>$ " equation (10) is a valid approximation for the Cherenkov spectral yield in the soft x-ray region in case transition radiation is also taken into account. For example, in case of a silicon foil and using $10-\mathrm{MeV}$ electron (see fig. $2 \mathrm{~b}$ ), the spectral yield calculated by equation (10) (Frank-Tamm approach) is equal to the spectral yield calculated by equation (17) (Ginzburg-Frank approach) on the non-absorbing site of the L-edge.

On basis of equation (18) the intensity difference between both radiation phenomena can be discussed. It is well known that transition radiation gives rise to the main intensity contribution in the $\mathrm{x}$-ray region for high-energy electrons at small angles. This is shown by the approximating of equation (18) for high-relativistic electrons $\left(\gamma^{-2}<<\chi^{\prime}\right)$, i.e.

$$
\frac{d N}{d \omega}=\frac{\alpha}{\omega \pi} \ln \left(\frac{\chi^{\prime}}{\gamma^{-2}}\right)+\frac{\alpha}{\omega} \frac{\chi^{\prime}}{\chi^{\prime \prime}} \eta\left(\chi^{\prime}\right)
$$

In equation (19) the spectral Cherenkov yield becomes constant for high-energy electrons, while the transition radiation part still increases with $\gamma$. For moderate electron energies (up to $25 \mathrm{MeV}$ ), however, the spectral transition radiation yield can be neglected to the spectral Cherenkov yield as can be seen from fig. $2 \mathrm{a}$ in case of silicon using 5 or $10 \mathrm{MeV}$ electrons.

\subsection{Soft x-ray Cherenkov radiation characteristics}

In this section the characteristics of soft x-ray Cherenkov radiation will be summarized by using equation (10) and by looking at the resonant anomalous dispersion (e.g. fig. 3a and 3b): (i) The Cherenkov radiation is characterized by a narrow bandwidth, because the spectral yield in equation (10) is large where $\chi^{\prime}-\gamma^{-2}>>\chi^{\prime \prime}$. Therefore, the spectrum from an absorbing material is dominated by the spectral part that fulfills the Cherenkov condition at the non-absorbing site of the edge. Typically, the Cherenkov radiation has a FWHM of a few eV. (ii) The Cherenkov radiation is concentrated within a narrow angular, forwardly directed profile, because the emission angles are small as can be seen from the approximation of equation (1)

$$
\theta=\sqrt{\chi^{\prime}-\gamma^{-2}}
$$

This angular profile is a hollow intensity distribution, because the spectral Cherenkov intensity (10) is proportional to the emission angle squared as can be seen by substituting equation (20) into equation (10). Typically, the maximum Cherenkov intensity is at an angle of a few degrees up to $10^{\circ}$.

For an absorbing medium, however, this fixed relation between refractive index value and emission angle does not strictly hold. Due to the fact that the emitted Cherenkov radiation originates from a finite path length, which is determined by the absorption length, the angle of constructive interference is not well defined. Therefore, the angular Cherenkov profile shows broadening due to this diffraction effect, which is taken into account by equation (15) and 
illustrated in fig. 2a. Despite this diffraction, the angular emission profile of Cherenkov radiation from an absorbing medium is still a hollow profile with the maximum intensity given by the Cherenkov angular relation.

So far, we have observed ${ }^{9}$ silicon L-edge $(99.7 \mathrm{eV})$ Cherenkov radiation generated by $5-\mathrm{MeV}$ electrons. We have analyzed the radiation using a crude spectrometer based on a Si/Mo-multilayer mirror and a photodiode placed in a $\theta-2 \theta$ configuration. These experiments showed that the observed total yield and dependence of the photon yield spectrum on emission angle are in agreement with theoretical predictions based on refractive index data of silicon. Just recently, we have observed ${ }^{19}$ for the first time Cherenkov radiation emitted in the water-window spectral region from a titanium and vanadium foil generated by $10-\mathrm{MeV}$ electrons. In the following section these measurements will be discussed in somewhat greater detail.
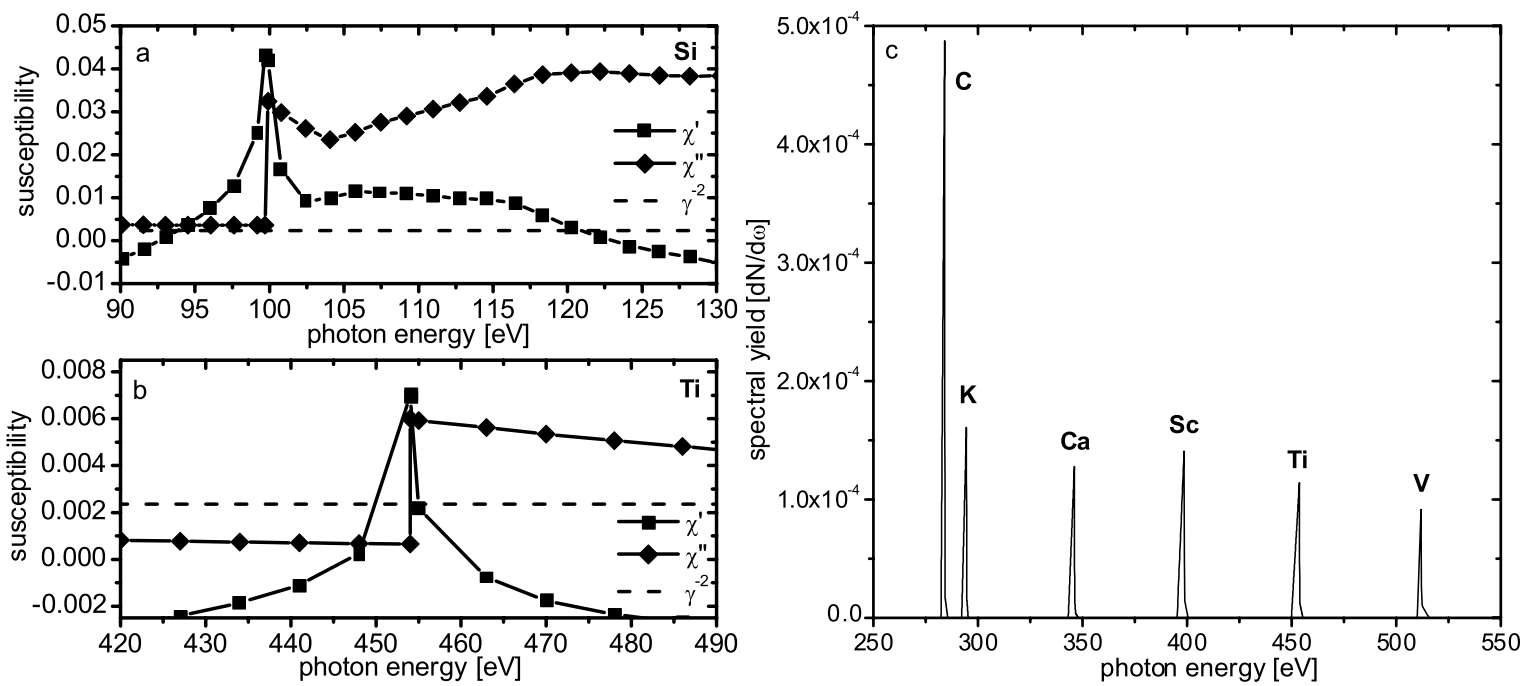

Figure 3: Susceptibility $\chi$ of respectively (a) silicon and (b) titanium at their L-edges. The dashed line indicates the Cherenkov condition for $10-\mathrm{MeV}$ electrons. (c) The theoretical Cherenkov spectral yield of different materials in the water-window spectral region using $10-\mathrm{MeV}$ electrons.

\section{WATER-WINDOW CHERENKOV RADIATION}

The water window is the spectral region between the carbon K-absorption edge $(284 \mathrm{eV})$ and the oxygen $\mathrm{K}$-absorption edge $(543 \mathrm{eV})$. This spectral region is ideal for $\mathrm{x}$-ray microscopy of biological samples due to the relative transparency of water and the high natural contrast. The special characteristics of soft x-ray Cherenkov radiation, which are discussed in the previous section, makes this source interesting for such applications. In fig. $3 \mathrm{c}$ the theoretical spectral Cherenkov yield is shown for materials that are Cherenkov emitters in the water window based on the refractive index data of ref. 14. For the first experiments, which are discussed in this section, titanium and vanadium are selected, because both metals are readily available in $10-\mu \mathrm{m}$ thick foils. This thickness is for both materials much larger than the absorption length (about $0.5 \mu \mathrm{m}$ ) at the Cherenkov photon energy. In section 3.1 the experimental setup is discussed. The spectra recorded by a photon counting, soft x-ray CCD camera are explained in section 3.2. From these absolute calibrated spectra of both materials angular intensity profiles of the Cherenkov radiation are derived, which are presented in section 3.3.

\subsection{Experimental setup}

The experimental setup is designed to analyze the emitted radiation as a function of emission angle. Because the emitted Cherenkov radiation has a cone-like intensity profile that is cylindrical symmetric around the electron trajectory it is sufficient to measure the profile in one plane. The angular range of the detection angle is limited to $-20^{\circ}$ to $+20^{\circ}$, because all the possible soft x-ray Cherenkov emitters will be well within this range. The distance from the Cherenkov 
emitter to the detector unit is fixed to $1 \mathrm{~m}$. In combination with a beam spotsize of $1 \mathrm{~mm}$, the angular resolution will be about $1 \mathrm{mrad}$. To make use of this high angular resolution the electron beam divergence should be less than $1 \mathrm{mrad}$. During the measurements presented in this paper, the angular resolution was lower, because the divergence was larger due to elastic scattering of the electron beam in these $10-\mu \mathrm{m}$ foils (see section 3.3).

The electrons are generated by a $10-\mathrm{MeV}$, traveling wave, linear accelerator (M.E.L. SL-75). This is a standard medical electron accelerator, which can operate from 9 to $11 \mathrm{MeV}$ depending on the accelerator current. This accelerator generates an electron beam with a typical energy spread of $4 \%$ and a transverse emittance (i.e. rms spot size times rms divergence) of about $10 \mathrm{~mm} \cdot \mathrm{mrad}$. After the electrons have passed through the Cherenkov emitter a $90^{\circ}$-dipole magnet bends the electron beam into a beam dump where the current is measured. This beam dump is made of a carbon bar to reduce the Bremsstrahlung intensity from the electrons.

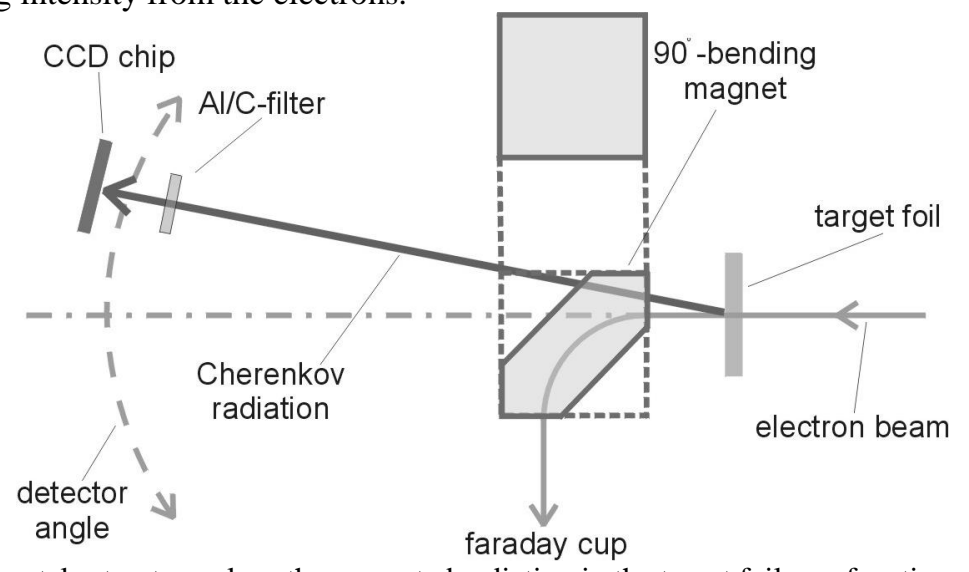

Figure 4: Experimental setup to analyze the generated radiation in the target foil as a function of emission angle.

The detector unit is a dedicated soft $\mathrm{x}$-ray CCD camera that is developed by Space Research Organization Netherlands for $\mathrm{x}$-ray astronomy. The CCD chip is a copy of the chips that are used in the European XMM-Newton satellite ${ }^{20}$. The camera is capable of measuring the energy of individual photons with a high quantum efficiency $(\approx 90 \%)$. Photon energies between $280 \mathrm{eV}$ and $15 \mathrm{keV}$ can be spectrally resolved. The FWHM-spectral resolution is $165 \mathrm{eV}$ at $1.5 \mathrm{keV}$ and scales with the square root of the photon energy. Therefore, the CCD chip is cooled to $-80^{\circ} \mathrm{C}$ to reduce the dark current. During the experiments the CCD camera is operated in the photon counting mode in which only $10 \%$ of the 800,000 pixels may be illuminated at any time. The average accelerator beam current was therefore set to a very low value of about $10^{9}$ electrons per second. In front of the CCD chip a thin aluminum-carbon filter (layer thickness of 150 $\mathrm{nm}$ and $27 \mathrm{~nm}$ respectively) with a diameter of $16 \mathrm{~mm}$ (Luxel corp.) is mounted to block any visible transition radiation from the foil. This limits the total detection solid angle to $2.2 \times 10^{-4} \mathrm{sr}$.

\subsection{Observed radiation spectrum}

When the electron beam passes through the foil, a few radiation phenomena can be observed. For example, in fig. 5a the pulse height spectrum of the detected radiation is shown generated by $10-\mathrm{MeV}$ electrons passing through $10-\mu \mathrm{m}$ foils of titanium, measured at an observation angle of $4^{\circ}$. In the pulse height spectra two peaks can be distinguished. A strong peak on the low-energy side, which is interpreted as Cherenkov radiation and the weaker peak on the high-energy side, which is identified as fluorescence radiation. The offset under the Cherenkov peak is a continuous background that decreases with photon energy, which is identified as transition radiation. The very small background around the fluorescence peak is Bremsstrahlung, which is mainly hard x-rays and is produced when the electrons pass through the foil and lose kinetic energy. By changing the detection angle the relative intensities of these radiation phenomena vary. At large detection angles the fluorescence radiation is dominant, because it has no angular dependence. This fluorescence radiation is used for the calibration of the CCD camera. At smaller detection angles Cherenkov and transition radiation and Bremsstrahlung are dominant. In the next section the specific Cherenkov angular profile will be discussed. In general, at small detection angles the total intensity on the CCD chip is larger than at large angles. Therefore, the average beam current has to be adjusted to get $10 \%$ of illumination at each detection angle. 

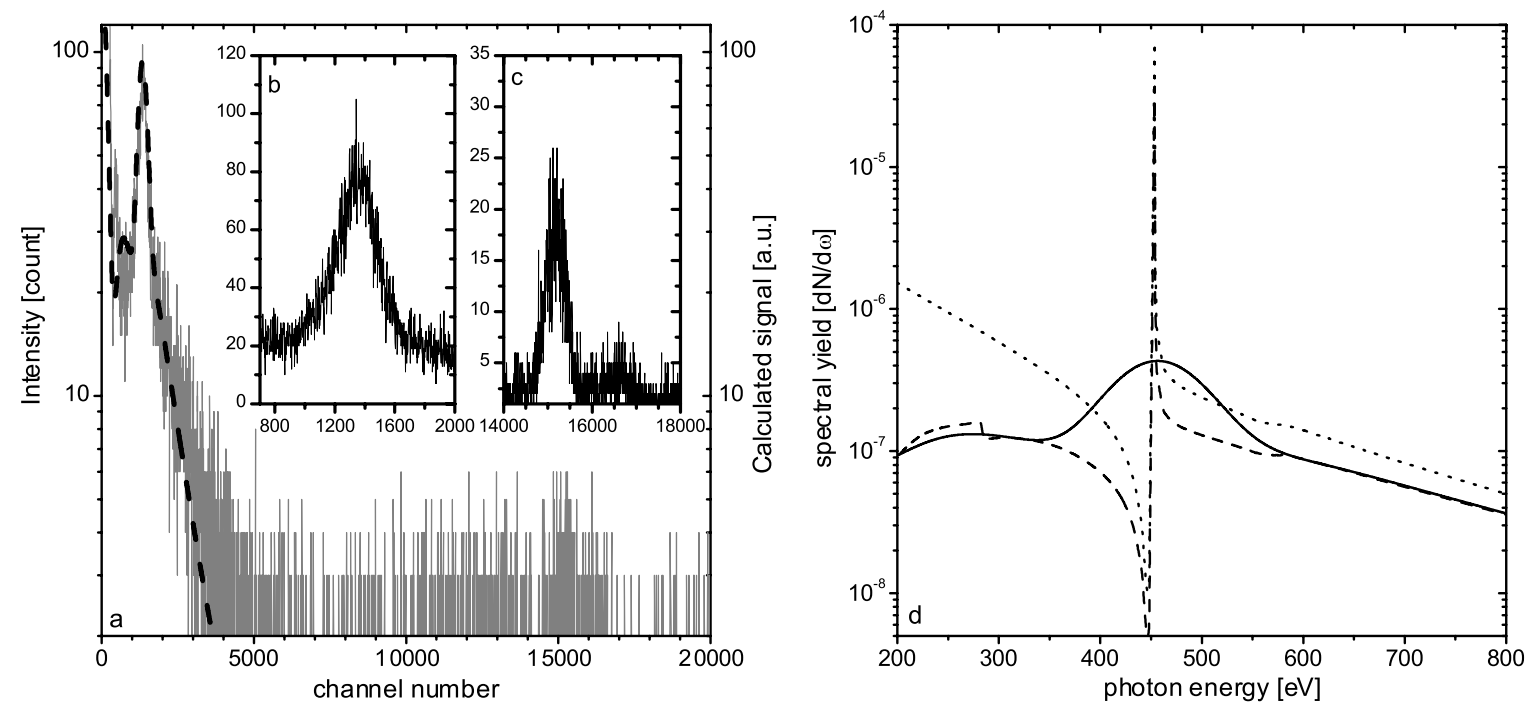

Figure 5: (a) Pulse height spectrum of the radiation produced by $10-\mathrm{MeV}$ electrons in titanium at an observation angle of $4^{\circ}$. The dashed curve is equal to the solid line in fig. 5d. (b) Enlargement of the Cherenkov peak. (c) Enlargement of the additional measurement of the fluorescence lines measured angle of $10^{\circ}$ using a higher electron beam current. (d) Theoretical calculation of the expected CCD spectrum starting from the spectral yield (dotted curve) and multiplied by the transition of $\mathrm{Al} / \mathrm{C}$-filter (dashed curve) and then taking the convolution with the CCD spectral response (solid curve).

The photon energy of the Cherenkov peaks can be obtained from the spectra by fitting the peaks to a Gaussian line profile with a linear offset. For the calibration of the CCD camera three $\mathrm{K}_{\alpha}$-fluorescence lines, which are produced by aluminum, titanium and vanadium foils, were used. The spectral resolution of the CCD camera was too low to resolve $\mathrm{K}_{\alpha 1}$ and $\mathrm{K}_{\alpha 2}$ from titanium and vanadium (see fig. 5c). Based on this calibration the Cherenkov peaks are experimentally determined at $459 \pm 2 \mathrm{eV}$ for titanium and $519 \pm 3 \mathrm{eV}$ for vanadium. A small, but significant, difference is observed with the theoretical peak positions at their respective L-edge, i.e. $453 \mathrm{eV}$ and $512 \mathrm{eV}$. The fact that we measure the peaks at slightly higher photon energies can be explained by a precise analysis of detection system as is shown for titanium in fig. $5 \mathrm{~d}$.

This analysis is started from the theoretical spectral radiation yield (equation (12)) that is integrated over the solid angle of the detector at an emission angle of $4^{\circ}$ (dotted curve). By multiplying this spectrum with the transmission of the $\mathrm{Al} / \mathrm{C}$ filter the spectrum is calculated that will incident on the CCD chip (dashed curve). Then, by convolving this spectrum with a spectral resolution of the CCD camera, i.e. $100 \mathrm{eV}$ at the titanium L-edge $(453 \mathrm{eV})$, the expected CCD camera spectrum is calculated (solid curve), which is also added as the dashed curve in fig. 5a. The peak position in this calculated CCD camera spectrum is determined at $458 \mathrm{eV}$. This shift is due to the fact that at a photon energy a few eV below the L-edge the real part of the refractive index is equal to unity and therefore both transition radiation and Cherenkov radiation are absent (see fig. 5d). The resulting dip shifts the peak in the convolution to slightly higher energies. The same analysis is repeated for vanadium. The peak position in the theoretical CCD camera spectrum is determined at $522 \mathrm{eV}$. The measured peak positions of both titanium and vanadium agree within the experimental error with the theoretical values obtained from the analysis.

The calculated spectra explain completely the low-energy part of the measured spectrum as is shown in fig. 5a for titanium. Notice that based on theory the Cherenkov peak of titanium has a FWHM width of $2.0 \mathrm{eV}$. Therefore, the amplitude of the Cherenkov peak is approximately 50 times higher than the measured peak in the spectrum in fig. $5 \mathrm{a}$. This leads to the conclusion that the radiation spectrum in the soft $\mathrm{x}$-ray region is completely dominated one intense peak of Cherenkov radiation. 

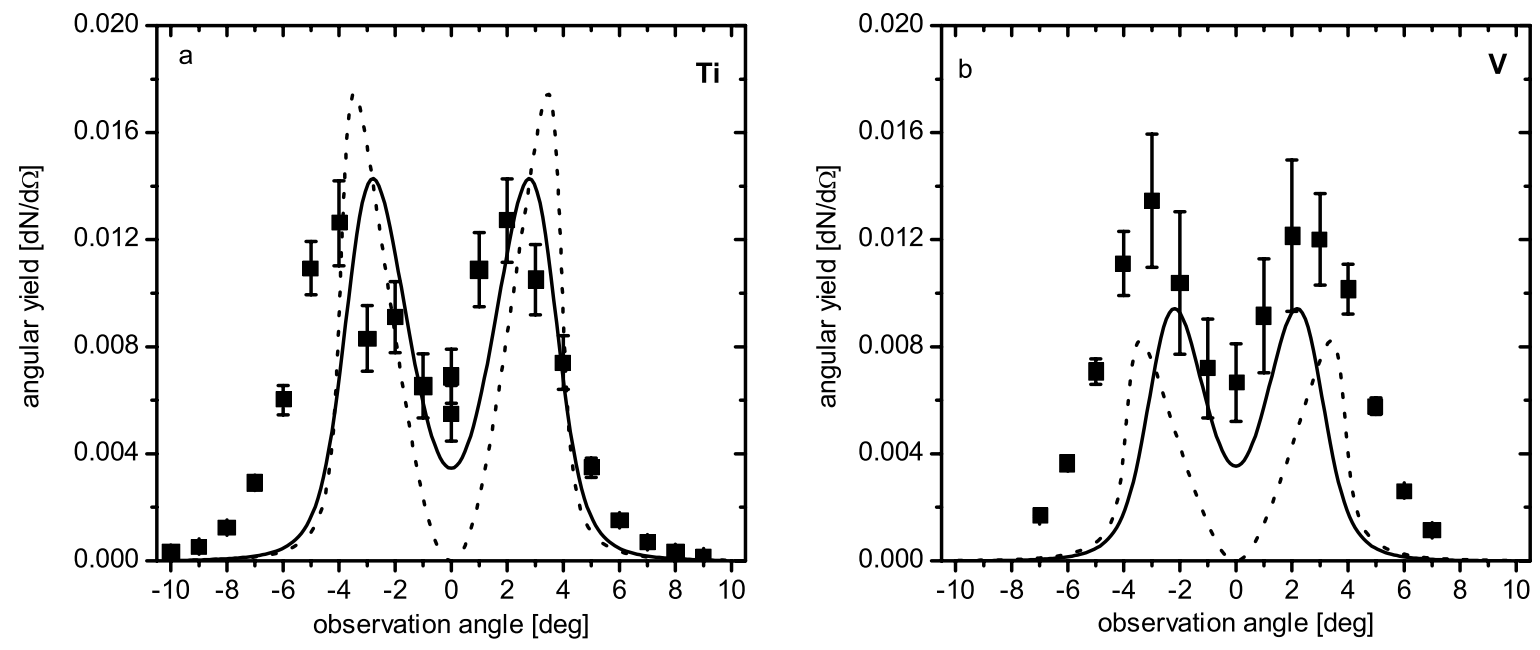

Figure 6: Measured Cherenkov angular yield [ph/(el- sr)] as a function of detection angle generated by 10-MeV electrons in titanium (a) and vanadium (b). The dotted curves are theoretical angular Cherenkov yields for an ideal parallel electron beam. The solid curves take elastic scattering of the electron beam in the foil into account as well.

\subsection{Angular profile and yield calculation}

Similar spectra as in fig. 5a have been obtained for both titanium and vanadium at observation angles ranging from $-10^{\circ}$ to $+10^{\circ}$. From each spectrum the area of the Cherenkov peak is determined, which gives the number of photons detected at each detector position. Then, by taking into account the quantum efficiency of the CCD camera, the transmission of the $\mathrm{Al} / \mathrm{C}$-filter, the solid angle of the detector and the number of electrons required to record a spectrum, the angular yield (number of photons per electron per solid angle) as a function of detection angle can be calculated, which is shown in fig. $6 \mathrm{a}$ and $6 \mathrm{~b}$. The measurement presented in fig. $6 \mathrm{~b}$ is improved compared to the one in ref. 19, due to an improvement in the quality of the electron beam. Clearly the typical Cherenkov angular profile is seen, i.e. a symmetric profile, which has a maximum associated with the maximum value of the refractive index $n$ and which rapidly drops to zero for larger angles. The angular profile is broadened due to the fact that the electrons are elastically scattered in the foil. We have analyzed this influence of elastic scattering of electrons in the foil on the angular Cherenkov emission profile by performing a small-angle, 2-D convolution of both electron and Cherenkov radiation angular distributions:

$$
f(\theta)=\int g\left(\theta^{\prime}\right) \theta^{\prime} d \theta^{\prime} \int h\left(\theta^{\prime \prime}\right) d \varphi^{\prime}
$$

in which $g(\theta)$ is the electron angular distribution and $h(\theta)$ is the Cherenkov radiation angular distribution. The angles are related by

$$
\theta^{\prime \prime 2}=\theta^{2}+\theta^{\prime 2}-2 \theta \theta^{\prime} \cos (\varphi)
$$

The electron distribution $g(\theta)$ is calculated by the Molière theory of multiple scattering ${ }^{21}$, that applies to the $10-\mu \mathrm{m}$ foils for which the average number of scatterings per electron is 42 for titanium and 56 for vanadium. The resulting scattering distributions have an average scattering angle of $1.1^{\circ}$ and $1.3^{\circ}$ respectively. The Cherenkov radiation distribution $h(\theta)$ (dotted curve in fig. 6a and 6b) is calculated by integrating equation (12) over the photon energies for which the Cherenkov condition is satisfied. In fig. $6 \mathrm{a}$ and $6 \mathrm{~b}$ the resulting photon distributions for the scattered electron beam are indicated by the solid curves. This broadening effect explains the non-zero angular yield value at $0^{\circ}$. The measured angular profile is also shifted with respect to the theoretical curve, which is probably due to a small misalignment of the electron beam.

Integrating the measured angular distribution over all emission angles we find a total yield of $3.5 \times 10^{-4}$ photons/el for titanium and $3.3 \times 10^{-4}$ photons/el for vanadium. For titanium this is slightly higher than the theoretical value of $2.4 \times 10^{-4}$ photons/el. For vanadium the experimental value is more than twice as high as the theoretical value of $1.4 \times 10^{-4}$ photons/el. In view of the very limited number of available data points, the measured spectra and angular profiles agree 
very well. The fact that the yield of vanadium is higher than theoretically expected, can be explained by assuming that the peak in the refractive index at the L-edge is slightly broader than according to the data of ref. 14. The higher yield of vanadium cannot be explained by a higher peak in the refractive index data, because this would give rise to a larger Cherenkov angle. The spectral resolution of the CCD camera is too low to determine the spectral line width that would have been an indication for this effect.

\section{CONCLUSIONS}

\subsection{Brightness discussion}

On the basis of the measured yields for titanium and vanadium, we can now evaluate the potential of a Cherenkovradiation-based compact source in the water window. Using a high-power, but laboratory-sized, $10-\mathrm{MeV}$ accelerator of $1-\mathrm{mA}$ average current the total output is $2.2 \times 10^{12}$ photons $/ \mathrm{s}(0.16 \mathrm{~mW})$ for titanium and $2.1 \times 10^{12}$ photons $/ \mathrm{s}(0.17 \mathrm{~mW})$ for vanadium. The corresponding brightness is $2.7 \times 10^{9}$ photons $/\left(\mathrm{s} \cdot \mathrm{mm}^{2} \cdot \mathrm{sr} \cdot 0.1 \% \mathrm{BW}\right)$ for titanium and $3.8 \times 10^{9}$ photons $/\left(\mathrm{s} \cdot \mathrm{mm}^{2} \cdot \mathrm{sr} \cdot 0.1 \% \mathrm{BW}\right.$ ) for vanadium, assuming a $100-\mu \mathrm{m}$ electron-beam spot size. These brightness values are comparable to the values obtained from laser-produced plasma ${ }^{2}\left(1 \times 10^{10}\right.$ photons $\left./\left(\mathrm{s} \cdot \mathrm{mm}^{2} \cdot \mathrm{sr} \cdot 0.1 \% \mathrm{BW}\right)\right)$ and highharmonic generation ${ }^{4}\left(5 \times 10^{7}\right.$ photons/(s. $\left.\left.\mathrm{mm}^{2} \cdot \mathrm{sr} \cdot 0.1 \% \mathrm{BW}\right)\right)$. We emphasize that in contrast to the laser-based sources, the Cherenkov spectrum consists of only a single, isolated peak and that no debris formation occurs.

\section{ACKNOWLEDGMENTS}

The authors thank Karin Hendriks for her contributions to the experiments and analysis, A.J. den Boggende and H. de Vroome of SRON (Utrecht, The Netherlands) for the technical assistance with the CCD camera and Jaap Corstens for the numerical evaluation of the 2-D convolution. This work was made possible by financial support from the Technology Foundation STW (The Netherlands) and is part of the research program of the Foundation for Fundamental Research on Matter (FOM, The Netherlands).

\section{REFERENCES}

1. C. Jacobsen, "Soft x-ray microscopy", trends Cell Biol. 9, 44-47, 1999.

2. G.A. Johansson, A. Holmberg, H.M. Hertz, and M. Berglund, "Design and performance of a laser-plasma-based compact soft x-ray microscope", Rev. Sci. Instr. 73, 1193-1197, 2002.

3. R. Lebert, G. Schriever, T. Wilhein, and B. Niemann, "Soft x-ray emission of laser-produced plasmas using a lowdebris cryogenic nitrogen target", J. Appl. Phys. 84, 3419-3421, 1998.

4. C.H. Spielmann, N.H. Burnett, S. Sartania, R. Koppitisch, M. Schnürer, C. Kan, M. Lenzner, P. Wobrauschek, and F. Krausz, "Generation of coherent x-rays in the water window using 5-femtosecond laser pulses", Science 278, 661-664, 1997.

5. Z. Chang, A. Rundquist, H. Wang, M.M. Murnane, and H.C. Kapteyn, "Generation of coherent soft x rays at $2.7 \mathrm{~nm}$ using high harmonics", Phys. Rev. Lett. 79, 2967-2970, 1997.

6. M. Wieland, R. Frueke, T. Wilhein, C. Spielmann, M. Pohl, and U. Kleineberg, "Submicron extreme ultraviolet imaging using high-harmonic radiation", Appl. Phys. Lett. 81, 2520-2522, 2002.

7. P. Rullhusen, X. Artru, and P. Dhez, Novel Radiation Sources Using Relativistic Electrons: From Infrared To XRays, World Scientific, Singapore, 1998.

8. V.A. Bazylev, V.I. Glebov, E.I. Denisov, N.K. Zhevago, M.A. Kumakhov, A.S. Khlebnikov, and V.G. Tsinoev, "Xray Cerenkov radiation. Theory and experiment", Sov. Phys. JETP 54, 884-892, 1981.

9. W. Knulst, O.J. Luiten, M.J. van der Wiel, and J. Verhoeven, "Observation of narrow-band Si L-edge Cerenkov radiation generated by $5 \mathrm{MeV}$ electrons", Appl. Phys. Lett. 79, 2999-3001, 2001.

10. C. Grupen, Particle Detectors, Cambridge University Press, Cambridge, 1996.

11. J.V. Jelley, Cherenkov radiation and its applications, Pergamon Press, London, 1958.

12. J.D. Jackson, Classical electrodynamics, Chap. 7, Wiley, Chichester, 1999.

13. D.Y. Smith and J.H. Barkyoumb, "Sign reversal of the atomic scattering factor and grazing-incidence transmission at X-ray-absorption edges", Phys. Rev. B 41, 11529-11535, 1990.

14. B.L. Henke, E.M. Gullikson, and J.C. Davis, "X-ray interactions: photoabsorption, scattering, transmission, and reflection at E=50-30000 eV, Z=1-92", At. Data and Nucl. Data Tables 54, 181-342, 1993. 
15. I. Frank, Ig. Tamm, "Coherent visible radiation of fast electrons passing through matter", C.R. Acad. Sci. USSR 14, 109-114, 1937.

16. B. Lastdrager, A. Tip, and J. Verhoeven, "Theory of Cerenkov and transition radiation from layered structures", Phys. Rev. E 61, 5767-5778, 2000.

17. M.L. Ter-Mikaelian, High-Energy Electromagnetic Processes in Condensed Media, Chap. 4, Wiley, Toronto, 1972.

18. V.L. Ginzburg and V.N. Tsytovich, Transition Radiation And Transition Scattering, Chap. 2, Hilger, Bristol, 1990.

19. W. Knulst, M.J. van der Wiel, O.J. Luiten, and J. Verhoeven, "High-brightness, narrowband and compact, soft x-ray Cherenkov sources in the water window", to be published

20. J.W. den Herder, et al., "The reflection grating spectrometer on board XMM-Newton", A\&A 365, L7-L17, 2001.

21. H.A. Bethe, "Molière's theory of multiple scattering", Phys. Rev. 89, 1256-1266, 1953 